\title{
Parasite Lost: Chemical and Visual Cues Used by Pseudacteon in Search of Azteca instabilis
}

\author{
Kaitlyn A. Mathis • Stacy M. Philpott • \\ Rayane F. Moreira
}

Revised: 21 December 2009 / Accepted: 10 November 2010 /

Published online: 1 December 2010

(C) The Author(s) 2010. This article is published with open access at Springerlink.com

\begin{abstract}
An undescribed species of phorid fly (genus: Pseudacteon) parasitizes the ant Azteca instabilis F Smith, by first locating these ants through the use of both chemical and visual cues. Experiments were performed in Chiapas, Mexico to examine a) the anatomical source of phorid attractants, b) the specific chemicals produced that attract phorids, and c) the nature of the visual cues used by phorids to locate the ants. We determined that phorid-attracting chemicals were present within the dorsal section of the abdomen, the location of the pygidial gland. Further experiments indicate that a pygidial gland compound, 1-acetyl-2-methylcyclopentane, is at least partially responsible for attracting phorid flies to their host. Finally, although visual cues such as movement were important for host location, size and color of objects did not influence the frequency with which phorids attacked moving targets.
\end{abstract}

Keywords Host location · alarm pheromone - dipteran parasitoids · coffee agroecosystems

\section{Introduction}

Parasitoids have evolved effective and efficient methods of host location, many of which involve utilization of chemical and visual cues. Social insects, such as ants, use chemical communication as a primary form of interaction along with some visual and tactile signals (Jackson and Morgan 1993; Hölldobler 1999). The

\footnotetext{
K. A. Mathis $(\bowtie)$

University of California, Berkeley, 137 Mulford Hall, Berkeley, CA 94720, USA

e-mail: Kamathis@berkeley.edu
}

S. M. Philpott

Department of Environmental Sciences, University of Toledo, 2801 W. Bancroft Street, Toledo, OH 43606, USA

R. F. Moreira

Department of Natural Science, Hampshire College, 893 West St., Amherst, MA 01002, USA 
chemical signals exchanged between ants are often complex and species specific (Jackson and Morgan 1993). The reliability of these intraspecific chemical signals thus makes them effective host location cues for ant parasitoids such as phorid flies (Brown and Feener 1991; Feener et al. 1996; Morehead and Feener 2000).

Many dipteran parasitoids in the family Phoridae use ants as hosts (Disney 1994). These phorid species are commonly referred to as the decapitating flies. In Pseudacteon species phorid flies that attack Solenopsis, the adult flies hover over ant hosts and dive down to oviposit an egg beneath the ant's exoskeleton. The larva hatches and moves through the ant into the head where it eats the contents and uses the empty shell as a case in which to pupate, eventually causing the ant's head to fall off (Cônsoli et al. 2001). Later, the adult phorid fly will emerge from the head of the dead ant to begin the cycle again (Porter 1998; Disney 1994). Although phorids have direct parasitic effects on ants (i.e. cause ant mortality), they also significantly change ant foraging behavior by limiting host resource acquisition behavior, modifying ant competitive hierarchies, and dampening ant effects on herbivores (Feener 1981; Feener and Brown 1992; Orr et al. 1995; Vandermeer et al. 2002; Philpott 2005b).

A number of different phorid-ant relationships have been described and, for each, it is generally reported that phorids use chemical and visual cues to locate ant hosts, but the specific cues used by phorid species to locate particular ants differ (Feener et al. 1996; Morehead and Feener 2000; Vander Meer and Porter 2002; Chen et al. 2009; Maschwitz et al. 2008; Gazal et al. 2009). Ants produce a range of pheromones with different functions in different glands throughout their bodies. These pheromones are responsible for behaviors such as trail marking, worker recruitment, and alarm signaling, each of which has been linked to attracting phorids. For example, the phorid parasitoid, Apocephalus paraponerae Borgmeier, is attracted to 4-methyl-3-heptanone and 4-methyl-3-heptanol produced in the mandibular glands of its host, Paraponera clavata Fabricius (Feener et al. 1996). The thoraces of ants contain the metaplural glands, which are the source of the kairomone known to attract Pseudacteon tricuspis Borgmeier to Solenopsis invicta Buren (Chen and Fadamiro 2007). The abdomens of ants also contain several glands that produce pheromones, although to our knowledge no compounds present within abdominal glands have previously been reported as phorid attractants.

The small amount of information reported on visual cues used by phorid flies indicates that these cues can also vary based on individual phorid-ant relationships. Some phorids oviposit on stationary hosts, whereas others require host motion (Wuellner et al. 2002). Other phorids select hosts based on host size or color (Owens and Prokopy 1986). For example, Apocephalus paraponerae attack stationary individuals preferring hosts with large body size (Moorehead and Feener 2000). Neodohrniphora curvinervis Malloch, a parasitoid of Atta cephalotes Linnaeus, also prefer larger worker castes as hosts (Orr 1992). Preference for larger hosts also occurs in Pseudacteon crawfordi Coquillett, a parasitoid of fire ants (however based on range phorid species might be Pseudacteon hippeus Plowes) (Feener 1987; Plowes et al. 2009).

One of the more recently described ant-phorid relationships is that between Azteca instabilis and an undescribed species of Pseudacteon. Azteca instabilis is an aggressive arboreal ant species ubiquitous over the New World tropics. On coffee 
plantations, these ants commonly create carton nests in the large shade trees and forage in the coffee plants surrounding the nest. Azteca instabilis tends scale insects living on coffee bushes and preys on other insect herbivores of the coffee leaves (Perfecto and Vandermeer 2006). The presence of A. instabilis within coffee plantations has a significant impact on the coffee agroecosystem as a whole and this species could be considered as a potential biological control agent of coffee herbivores (Vandermeer et al. 2002; Vandermeer et al. 2008). Presence of Pseudacteon phorid flies significantly decreases $A$. instabilis recruitment to resources, affects competitive interactions with other ant species, and may affect the ability of $A$. instabilis to control herbivore populations (Philpott et al. 2004; Philpott 2005a).

A substantial amount of effort has gone into examining the ecology and chemistry of Pseudacteon-ant relationships. Though Pseudacteon spp. have varying degrees of host specificity, some phorid species specialize on closely related ant species (Porter et al. 1995; Gilbert and Morrison 1997; Morrison and Gilbert 1999) With increased host specificity in the phorid-ant relationship, the chemical and visual cues used in host location are also likely to be more specific. Based on previous descriptions of the chemical ecology of ant-phorid relationships, at least two abdominal glands are putatively important sources of pheromones in A. instabilis. Pavan's gland, located on the ventral side of the abdomen between the 7th and 8th sternites, is the source of trail pheromones. The pygidial gland, located on the dorsal side of the abdomen between the 7th and 8th tergites, is significantly larger and contains the pungent alarm-defense pheromones of the A. instabilis (Do Nascimento et al. 1998). Three cyclopentyl ketones in their respective concentrations have been reported to be present in the pygidial glands of A. instabilis (Fig. 1a): 2-methylcyclopentanone (I), cis-1-acetyl-2-methylcyclopentane (II) and 2-acetyl-3-methylcyclopentene (III) (Wheeler et al. 1975) (Fig. 1a). However, practically nothing is known about how Pseudacteon phorid parasitoids orient towards A. instabilis, or the specific chemical or visual cues driving this relationship.

To amend this lack of information, we examined the chemical and visual cues acting to attract Pseudacteon phorids to A. instabilis. First we aimed to isolate the anatomical source of pheromones used by Pseudacteon parasites of $A$. instabilis by isolating glandular extracts from ant workers, presenting the extracts near $A$. instabilis nests, and observing phorid behavior. Second, using field experiments, we examined

Fig. 1 Compounds present in the pygidial gland of Azteca instabilis (a): 1methylcyclopentane (I), (cis)1-acetyl-2-methylcyclopentane (II), and 2-acetyl-3methylcyclopentene (III) and the synthesis of commercially unavailable 1-acetyl2-methylcyclopentane from 1-acetyl-2-methylcyclopentene (IV) (b)

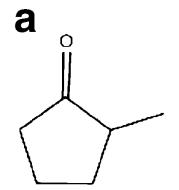

(I)

(IV)

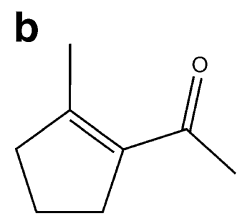

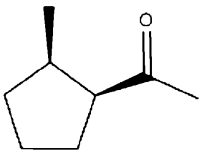

(II)

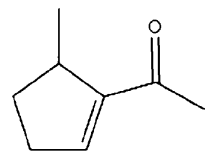

(III)

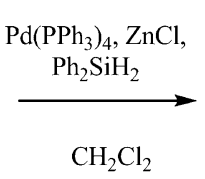

$\mathrm{CH}_{2} \mathrm{Cl}_{2}$

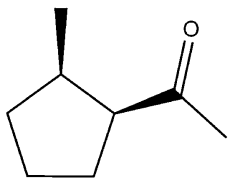

(II) 
whether pheromones from the aforementioned abdominal glands attract Pseudacteon parasitoids to A. instabilis, or whether glands from other body parts provide the necessary chemical cues. We were then able to obtain and make compound I and II respectively and examined whether one or both act as phorid attractants. Finally, we investigated the extent to which phorids use visual cues to locate and oviposit on $A$. instabilis ants by examining the importance of visual cues such as size, shape, and color in phorid host choice using life-size moving ant models.

\section{Methods}

\section{Study Site}

Field work was conducted in the wet season between 27 June and 29 July 2007, and in the dry season between 7 and 22 February 2008 on a shaded organic coffee farm, Finca Irlanda, in the Soconusco region of Chiapas, Mexico $\left(15^{\circ} 11^{\prime} \mathrm{N}, 92^{\circ} 20^{\prime} \mathrm{W}\right)$. The farm is located between 950-1,150 m elevation, receives approximately $4,500 \mathrm{~mm}$ of precipitation per year, and contains more than 200 species of shade trees. Mean temperatures during the dry season (May-August) range between $19^{\circ} \mathrm{C}$ and $25^{\circ} \mathrm{C}$ (Lin 2007). There are more than 60 arboreal ant species that occur in the farm, but $A$. instabilis is the most frequently encountered on the trunks of canopy trees (Philpott 2005b). Azteca instabilis builds carton nests on the tree trunks and in the lower canopy of the trees, and across the landscape, colonies of $A$. instabilis are distributed in patches (Vandermeer et al. 2008).

\section{Colony Collection and Description}

We collected individuals from four colonies of $A$. instabilis from areas of the farm under similar conditions and maintained these partial colonies in the laboratory for use in making body part extracts. We chose strong colonies with large sections of accessible carton for collection. In order to collect the nest, we cut as much of the carton as possible away from the tree and placed it into large plastic boxes, the rims of which were painted with INSECT-a-SLIP (BioQuip Products, Inc., Rancho Dominguez, 90220, CA). We collected individuals from two colonies on 27 June and the others were collected on 4 July. We collected individuals and nest material from colonies separated by a minimum of $100 \mathrm{~m}$ to ensure each were different colonies, and not satellite nests formed by budding. To confirm the independence of each lab colony, we placed individuals from each colony directly into tubs containing others and observed any aggressive behavior.

We provided all colonies with a coffee sapling (potted and under a meter in height) colonized by scale insects to tend, along with water, tuna, and sugar as needed. Each of the lab colonies varied in worker size, number of individuals, and in presence of reproductive individuals. Colony I was the smallest collected colony with only the smallest caste size of workers (head widths of workers are approximately $1.1 \mathrm{~mm}, 1.4 \mathrm{~mm}$, and $2.1 \mathrm{~mm}$ for small, medium, and large workers respectively); no male or female alates were found within the collected carton. Colonies II-IV contained all worker caste sizes as well as males and winged females. 
At the end of the summer field season, all individuals from colony I and II and the majority of individuals from III and IV were collected in vials, the vials were then filled with hexanes and placed in the freezer. These preserved ants were then used in the winter field season for preparation of whole body extracts. Vouchers specimens of Azteca instabilis and the Pseudacteon phorid flies have been deposited at El Colegio de la Fontera Sur (ECOSUR) in Tapachula, Mexico as well as the University of Toledo in Toledo, $\mathrm{OH}$.

\section{Preparation of Extracts}

To prepare head, thorax and abdomen extracts, ants were collected from each colony and frozen until dead. The ants were then trisected into head, thorax and abdomen sections with razor blades. Fifty heads, thoraxes, and abdomens were placed in separate 2-dram glass vials with $2 \mathrm{~mL}$ of pesticide-grade hexanes and crushed. For each colony, three extracts of heads, thoraxes and abdomens were prepared for a total of 12 extracts of each type. To prepare the dorsal and ventral abdominal extracts, 10 individuals were frozen and their abdomens were bisected under water. The dorsal and ventral segments were then placed in separate vials, each containing $2 \mathrm{~mL}$ of hexanes. When 10 abdomen sections were in each vial, the contents were pulverized. Colonies II, III, and IV alone were used during these trials as the workers in colony I were too small to accurately section the abdomens with available equipment. Three extracts of each body section were prepared from each colony. In preparation of whole body extracts, 50 previously frozen ants from the colonies were added to a 2-dram glass vial with $1.5 \mathrm{~mL}$ of hexanes and crushed.

Compounds found within the glands of $A$. instabilis as well as another general ant alarm compound were diluted and prepared to use for observations with phorid flies. Extracts of 2-methyl-cyclopentanone (I) were prepared using commercially available 2 -methyl-cyclopentanone in a $0.17 \%$ by volume solution with hexanes $(1.5 \mathrm{~mL})$. The formic acid extract was prepared in a similar manner. 1-acetyl-2-methyl-cyclopentane (II) was prepared from commercially purchased 1-acetyl-2-methyl-cyclopentene by reduction of the double bond (Fig. 1b). Diphenylsilane (1.55 mol eq.), anhydrous $\mathrm{ZnCl}_{2}$ (0.40 mol eq.), and tetrakis triphenylphosphine palladium (1.8 mol\%) were added to IV (60 mg) and $\sim 2 \mathrm{~mL}$ of chloroform. The reaction was sealed at room temperature and allowed to stir overnight. The mixture was purified using column chromatography on silica gel give a final yield of $\sim 30 \%$. The identity of the final product, II, was confirmed by GC/MS and ${ }^{1} \mathrm{H}$ NMR.

\section{Preparation of 3-D Model of Swarming Ants}

To have the capacity to experimentally isolate chemical from visual cues as phorid attractants, we prepared a 3-D model of swarming ants to simulate ant movement without chemical signals. This model consisted of magnets $(10 \times 4 \mathrm{~mm})$ placed on a platform itself sitting atop a magnetic conveyor belt made from bike chain and powered by a servo motor charged by a $6 \mathrm{~V}$ battery (Fig. 2). When in motion, the magnets move in a pattern that gives the effect of ants swarming in many directions. To examine visual cues necessary for host location and oviposition, we prepared two different types of model ants to place on the magnets and magnetic platform to attract 

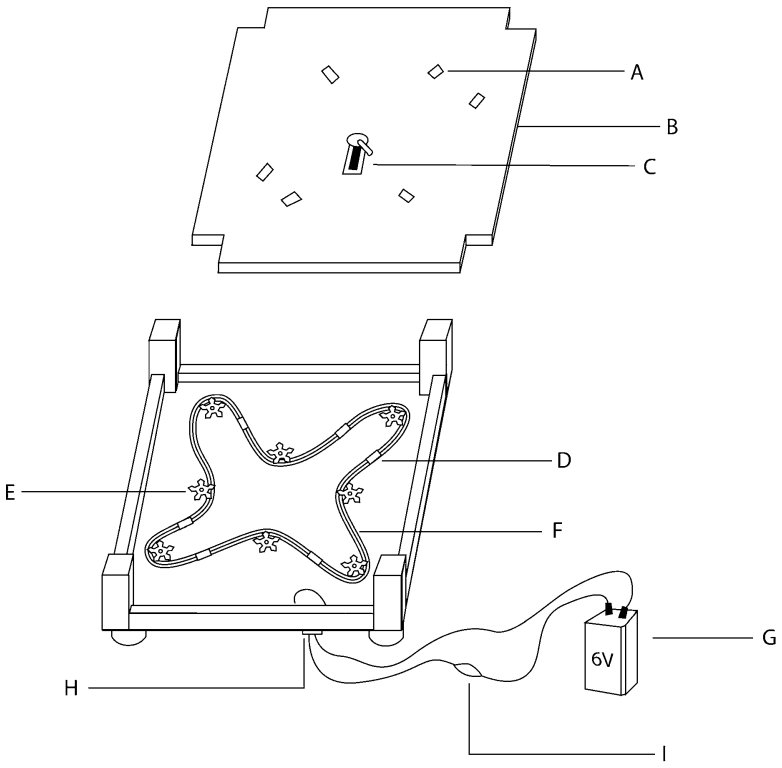

Fig. 2 Schematic diagram of the 3-D swarming ant model. $A$ : Magnets (with or without clay ants/dead $A$. instabilis) observed for phorid attack. $B$ : The platform the magnets move across in a seemingly random pattern. During trials the platform rests directly on top of the rest of the mechanism. $C: 2$ dram screw cap vial containing 50 crushed $A$. instabilis workers in hexanes and a filter paper wick. $D$ : The other half of the magnets attached to the bike chain beneath the platform. $E$ : The sprockets that guide the bike chain as it is propelled by the motor. $F$ : The bike chain. $G$ : $6 \mathrm{~V}$ battery used as a power source for the motor. $H$ : Servo Motor. I: Dial that will increase or decrease resistance, effectively acting as a power switch as well as allowing the operator to control the speed of the ant model magnets

phorids. We used freshly killed $A$. instabilis and ant-shaped clay models on the platform of our model. Ants used in visual cue observations were collected fresh from A. instabilis nests and frozen until dead. To examine whether size of host matters for Pseudacteon parasites of $A$. instabilis, we collected individuals from the largest $(5 \mathrm{~mm})$, middle $(3-4 \mathrm{~mm})$ and smallest $(<3 \mathrm{~mm})$ caste size for our experiments. We then washed these ants with hexanes to remove cuticular hydrocarbons or any other chemical cues that may be of further use to the phorids in host location. Once dry, we attached the ants to rectangular magnets using gorilla glue (The Gorilla Glue Co., 45227, OH). To examine whether color is an important visual cue for Pseudacteon parasites of A. instabilis, we molded red, yellow and brown ant shapes (approximately $5 \mathrm{~mm}$ in size) out of Fimo dough (Eberhard Faber, 91311, CA) and glued them to small square magnets to make ant models for color choice tests. Azteca instabilis ants most closely match the red color used in clay models. We prepared fresh versions of these models every day this type of observation was performed.

\section{Phorid Observations}

During the summer field season, we placed extracts made from different $A$. instabilis body parts near $A$. instabilis nests to record phorid attraction. Twelve strong $A$. instabilis colonies, defined as those with between 5-30 workers foraging at the base 
of the nest, each separated by at least $30 \mathrm{~m}$ were used as trial sites. To deliver pheromone into the air surrounding the nest, we placed open vials containing the prepared extract, with a filter paper wick, near the base of the nest. We presented each of the five extract types (head, thorax, abdomen, ventral abdominal section, dorsal abdominal section) on different days to prevent any potential contamination or any possible synergistic effects due to the presence of multiple compounds. On a sixth day, wicks doused in solvent controls were presented at A. instabilis colonies. To prevent differences between ant activities at each observation site from affecting the outcome, each field colony was visited each day at approximately the same time, and in the same order. The source of the extracts (i.e. lab colony) presented to each colony was randomized to account for any possible differences in extracts (concentration, composition) produced by individual lab colonies.

After presenting extracts to the air, the area surrounding the vials (approximately $30 \mathrm{~cm}^{2}$ ) was observed for $10 \mathrm{~min}$ and any phorid attack sessions were recorded. The phorid flies tend to attack by hovering over a moving ant, then diving at the ant between approximately 1 and 30 times before leaving the trial site. We considered independent phorid attack sessions to be any time in which the phorid fly approached the vial, then hovered over a single ant and dove toward the ant at least once. Multiple attacks on the same ant directly after one another were counted as the same attack session.

During the winter field season, we performed 15 min observations with extracts of whole body A. instabilis, along with formic acid, compound I, and compound II (each $0.17 \%$ by volume in $1.5 \mathrm{~mL}$ hexanes). Due to relative availability of the extract types, sample size differed for each of the extracts. We observed eleven replicates with compound I, nine with formic acid, six using compound II, six with and whole body extract. Otherwise, observation methods were the same as described above.

To examine visual cues used by phorids, we placed the 3-D swarming ant model within one meter of a strong colony of $A$. instabilis. We then added to the center of the platform, 6 model ants (either two of each color (yellow, brown, and red) or two of each size (small, medium, and large), along with a vial containing extract prepared from whole bodies of $A$. instabilis (Fig. 2). Extract preparation and set up of wires required several minutes during which the model ants remained stationary on the platform and vials with extracts remained closed. It is important to note that no phorid flies appeared until the extract of 50 crushed workers was opened and no flies attacked a model until the motor was turned on. To begin a 15 min observation period, we turned on the motor after opening the extract vial and inserting the filter paper wick. During observations, we recorded the number of phorid attack sessions on each ant type. As a control, we placed 6 magnets without ant models on the platform, along with the whole body extract and observed the number of phorid attack sessions on moving magnets for $15 \mathrm{~min}$. Additional control observations were performed with each model type where the models remained stationary throughout the 15 min observation period. We performed 12 replicates each of type of visual cue choice (size, color, and control observations as well as a duplicate set of no movement control observations).

\section{Statistics}

The five extract types as well as the abdominal extracts were compared using a Chisquare test of proportions. Additionally, the number of phorid attack sessions for 
each chemical and visual cue was compared using univariate ANOVAs and Tukey post hoc tests. We used ANOVA to compare the number of phorid attack sessions depending on a) chemical cues presented to phorids, b) size of visual cues, c) color of visual cues, and d) type of visual cue (dead ant, clay, control). In order to meet conditions of normality, all data were log-transformed (ln $(1+$ number of phorid attack sessions)).

\section{Results}

\section{Chemical Cues}

Of the five anatomical extracts and solvent control presented at $A$. instabilis colonies, only the abdomen extract attracted phorid flies (Table 1). Moreover, the extracts prepared from the dorsal portion of the abdomen attracted more phorid flies than extracts prepared from the ventral portion. These results suggest that the chemical attractant(s) is located within the dorsal portion of the abdomen (ChiSquare $=268, d f=4, P=0.001$ ).

Two compounds known to be present within the pygidial glands of $A$. instabilis were then tested. While compound I did not attract phorid flies, compound II attracted phorid flies. However, whole body extracts of $50 \mathrm{~A}$. instabilis attracted significantly more phorid flies than the 1-acetyl-2-methylcyclopentane (II) extracts $(F=25.774, d f=3, P=0.001$; Fig. 3). During trials with compound II a small percentage of foraging ants $(<5$ on average) were observed aggregating near the foreign vial and attacking it. To ensure the potential alarm responses of these foraging ants were not attracting phorids, formic acid extracts were also presented at trial sites to disturb the ants and induce an alarm response. These extracts attracted a slightly larger number of foraging $A$. instabilis (approximately $7-10$ on average) that attacked the vials in a similar manner as those in compound II trials. However, no phorid flies were observed during the trials with formic acid, indicating that phorids

Table 1 Total number of phorid flies attracted to head, thorax, abdomen, dorsal abdomen section and ventral abdomen section extracts of $A$. instabilis as well as whole uncrushed ants in hexanes and a hexane control. Total number of trials $=84$

Total number of attack sessions per extract type and colony number

\begin{tabular}{lccrrr}
\cline { 2 - 5 } Extract type & Colony 1 & Colony 2 & Colony 3 & Colony 4 & Total \\
\hline Hexane Control & 0 & 0 & 0 & 0 & 0 \\
Whole Uncrushed Ants in Hexanes & 0 & 0 & 0 & 0 & 0 \\
Heads & 0 & 0 & 0 & 0 & 0 \\
Thoraxes & 0 & 0 & 0 & 0 & 0 \\
Abdomens & 23 & 16 & 3 & 14 & 67 \\
Dorsal Portion of Abdomens & - & 14 & 0 & 0 & 36 \\
Ventral Portion of Abdomens & - & 1 & & 19 \\
\hline
\end{tabular}


Fig. 3 Average number of phorid attack sessions during trials using 1-acetyl2-methyl-cyclopentane, 2-methylcyclopentanone, formic acid, and whole body extracts. Bars with the same letters are not significantly different at $P=0.05$ )

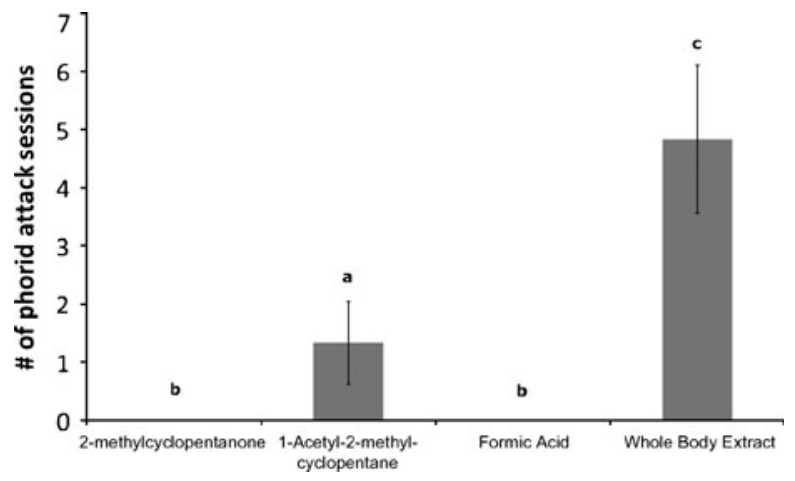

observed during compound II trials were attracted by compound II alone. Ants were observed to assume defensive postures due to presentation of both 1-acetyl-2methylcyclopentane and formic acid, but no phorid flies were observed during the formic acid extract observations, indicating that presence of phorids during 1-acetyl2-methylcyclopentane observations was due to the extract alone.

\section{Visual Cues}

Phorid flies did not attack the models when stationary and only a very small number of phorids attacked the moving magnets alone (Fig. 4a). This indicated that these phorid flies need some type of visual cue resembling their host ant to orient and attack. However, size and color do not appear to be critical components of the visual cue (Fig. 4b, c). During our observations, numbers of phorid fly attacks on the clay model ants as well as the dead A. instabilis did not differ, but phorids attacked models more than 10 times more often that magnets alone $(F=4.842, d f=2, P<0.03)$. However, the phorid flies had no significant preference with respect to size of dead ants $(F=0.783, d f=3, P>0.5)$ or color of clay models $(F=0.071, d f=2, P>0.9)$.

\section{Discussion}

\section{Anatomical Extracts}

Our results give strong evidence that this species of Pseudacteon uses pheromones produced in the dorsal portion of A. instabilis abdomens as host location cues. In only one trial out of a total of 9 observations, a ventral extract attracted a single phorid fly. This outlier is likely the result of a contamination of the extract during the abdomen bisection rather than resulting from trail compounds within the Pavan's gland attracting phorids to their hosts. Overall, the abdominal extracts attracted a larger number of phorid flies per trial than the dorsal extracts; however, abdomen extracts contained 5 times more $A$. instabilis individuals than the extracts made from dorsal sections of the abdomens alone. This result possibly indicates a correlation between $A$. instabilis and phorid fly densities, consistent with previous studies (Vandermeer et al. 2008; Philpott et al. 2009). 

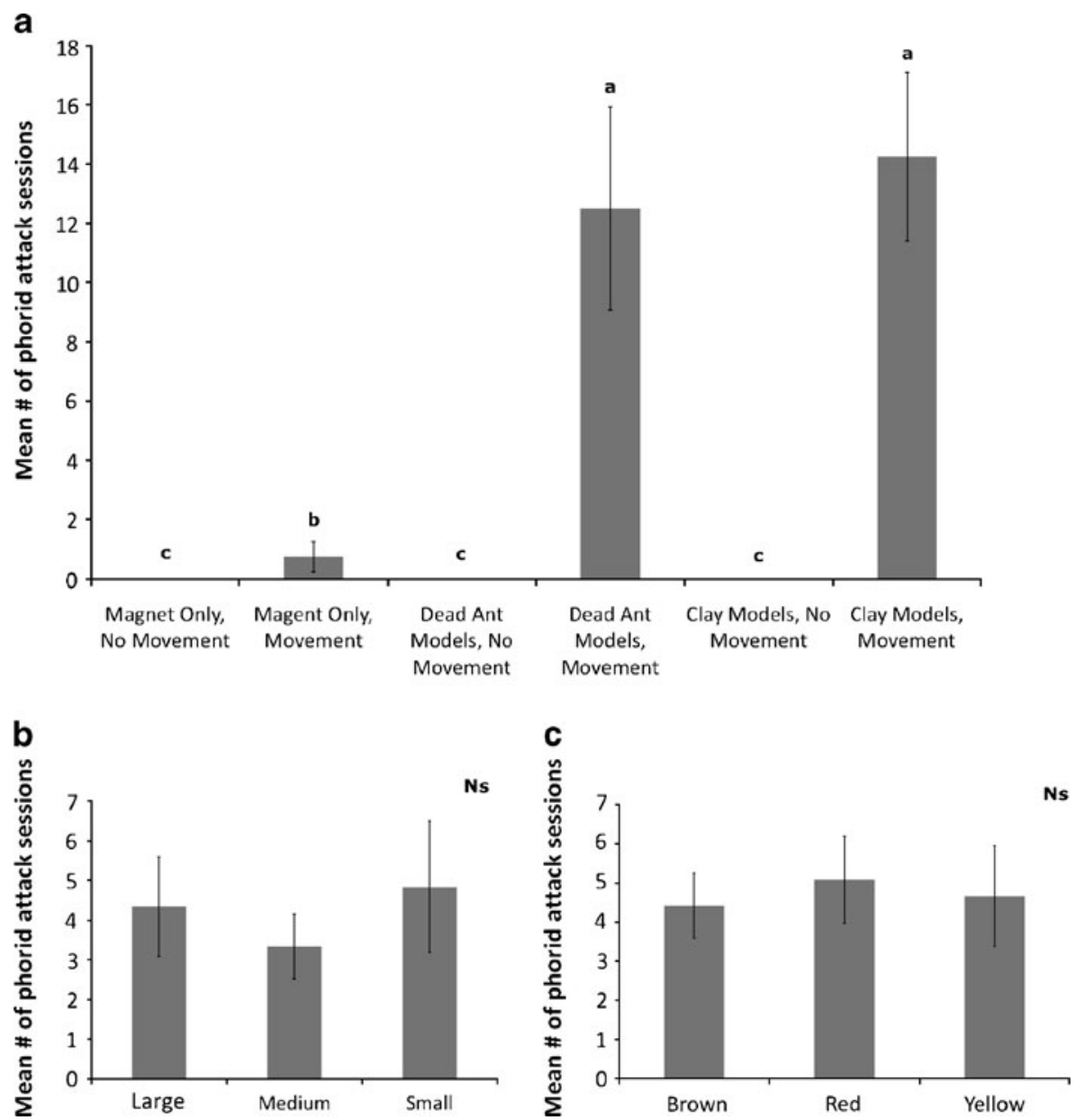

Fig. 4 Average number of phorid attack sessions on different visual cues presented to Pseudacton phorids at Azteca instabilis colonies. Phorid responses were compared based on type of cue presented both with and without movement of the models (a), size of visual cue (b), and color of visual cue (c). Bars with the same letters are not significantly different at $P=0.05$ )

Alarm-defense pheromones are emitted from the pygidial gland of Azteca spp. ants (Wheeler et al. 1975). Furthermore, the use of alarm-defense pheromone in phorid host location is consistent with observations at Finca Irlanda. The alarm-defense pheromones of $A$. instabilis, very easily triggered by nest disturbance, smell strongly of blue cheese, thus it is easy to detect whether the ants have emitted alarm-defense compounds. Phorid flies are far easier to locate near an A. instabilis nest after the nest has been disturbed in some way (generally by blowing on the nest or poking it with a stick). Within seconds of nest disturbance the characteristic odor of $A$. instabilis can be detected and often within minutes multiple phorid flies can be seen attacking the swarming $A$. instabilis. Furthermore, in other studies with phorid flies and $A$. instabilis, phorids are observed attacking their host species at tuna baits. This observation is also potentially consistent with our data as baits are generally forums for competitive interactions between A. instabilis and other species of ants (LeBrun and 
Feener 2002; Orr et al. 2003; Morrison and King 2004; Philpott 2005b). These interspecific interactions may induce the production of alarm-defense pheromones, thus attracting phorid flies to baits with multiple ant species competing for resources.

\section{Chemical Extracts and Whole Body Extracts}

While cis-1-acetyl-2-methyl-cyclopentane (II) was found to attract phorid flies, the relative abundance of phorid attack sessions was small when compared to observations using whole body extracts. This result could be an effect of relative concentration of the chemical compound used or it may be possible that another compound within the pygidial gland of $A$. instabilis is also an important attractant of phorid flies. Apocephalus paraponerae, for example, are attracted to a mixture of 4methyl-3-heptanone and 4-methyl-3-heptanol in a 9:1 ratio (Feener et al. 1996; Moorehead and Feener 2000). Furthermore, phorid flies were not attracted to model ants where formic acid was presented although nearby foraging ants became visibly alarmed with the use of both the formic acid and compound II. To control for alarm responses of foraging ants caused by alarm-defense compound extracts, formic acid extract was tested at trial sites. Though foraging ants produced a typical alarm response, it was not strong enough to attract phorid flies during the 15 min trials. Therefore while foraging ants at trial sites with compound II were producing alarm responses, it is unlikely that these alarm responses were responsible for phorid presence.

Movement, Size, Shape and Color Cues

Other species of Pseudacteon are reported to use a hierarchy of chemical and visual cues in host location (Moorehead and Feener 2000). While this Pseudacteon sp. appears to require motion and an approximately ant shaped form in order to hover over and attack a host, these phorids do not appear to be very specific as to what they will attack as long as the correct chemical attractant is present. Phorids, like many other taxa, thus require multisensory (chemical and visual) cues to locate hosts (Partan and Marler 2002). Phorids were as almost equally as likely to attack any of the clay models, indicating that color is not a crucial factor in host choice. Ant models of the smallest, middle and largest caste sizes were all equally attacked as well. Moreover the clay models, although less realistic, were as effective as the dead ant models at attracting flies. Indeed, clay models had slightly more phorid attack sessions than the dead $A$. instabilis individuals. In order to fully understand the visual cues used by this species of Pseudacteon, other aspects such as speed of the host should be taken into consideration. The speed of the ant models on the conveyer belt was kept constant in the experiment, but could be varied in future studies.

\section{Implications}

A substantial amount of effort has gone into examining the relationship between fire ants and their Pseudacteon parasitoids with the hope that phorid flies will be an effective biological control agent against this invasive species (Chen and Fadamiro 2007; Feener and Brown 1992; Morrison and Gilbert 1999; Morrison and King 
2004; Morrison and Porter 2006). In the case of $A$. instabilis, however, a high level of foraging activity is important in coffee agroecosystems where $A$. instabilis acts as a biological control agent for coffee pests. A better understanding of the chemicals attracting Pseudacteon to A. instabilis may potentially elucidate whether these compounds could be utilized as a bait in phorid traps placed on coffee farms to ease the effects of phorid flies on $A$. instabilis foraging and survival in order to promote the ant's defense of the coffee plants. Similar traps have been designed for other systems (Puckett et al. 2007). However, further investigation into the nature of the chemical attractants such as required concentration of cis-1-acetyl-2-cyclopentane, information on whether compound III or any other additional compound in the pygidial gland works as a synergistic attractant, and a better understanding of the distance with which phorid flies are able to detect this compound (or potential compounds) is needed before baited phorid traps could be effectively constructed.

Acknowledgments We thank G. López Batista, G. Dominguez, B. Esteban Chilel, and A. de la Mora Rodriguez for assistance with nest collection along with R. Mesch and V. Finnan for help in the lab. We thank C. Lentz for assembling the swarming ant model as well as Bernardo and Walter Peters for granting us permission to work at Finca Irlanda. Also thanks to B. Schultz, M. Feinstein, S. Partan, and for providing helpful comments. This research was funded in part by NSF grant DEB 0349388 to J. Vandermeer and I. Perfecto, The University of Toledo, and the Ray Coppinger Fund. These experiments comply with the current laws of Mexico, the location in which they were performed.

Open Access This article is distributed under the terms of the Creative Commons Attribution Noncommercial License which permits any noncommercial use, distribution, and reproduction in any medium, provided the original author(s) and source are credited.

\section{References}

Brown BV, Feener DH (1991) Behavior and host location cues of Apocephalus paraponera (Diptera: Phoridae), a parasitoid of the giant tropical ant, Paraponera clavata (Hymenoptera: Formicidae). Biotropica 23:182-187

Chen L, Fadamiro HY (2007) Behavioral and electroantennogram responses of phorid fly Pseudacteon tricuspis (Diptera: Phoridae) to red imported fire ant Solenopsis invicta odor and trail pheromone. J Insect Behav 20:267-287

Chen L, Sharma KR, Fadamiro HY (2009) Fire ant venom alkaloids act as key attractants for the parasitic phorid fly, Pseudacteon tricuspis (Diptera: Phoridae). Naturwissenschaften 96:1421-1429

Cônsoli FL, Wuellner CT, Vinson SB, Gilbert LE (2001) Immature development of Pseudacteon tricuspis (Diptera: Phoridae), an endoparasitoid of the red imported fire ant (Hymenoptera: Formicidae). Ann Entomol Am 94:97-109

Disney RHL (1994) Scuttle flies: the phoridae. Chapman \& Hall

Do Nascimento RR, Billen J, Stant'ana AEG, Morgan ED, Harada AY (1998) Pygidial gland of Azteca NR. bicolor and Azteca chartifex: Morphology and chemical identification of volatile components. J Chem Ecol 24:1629-1637

Feener DH (1981) Competition between ant species-outcome controlled by parasitic flies. Science 214:815-817

Feener DH (1987) Size selective oviposition in Pseudacteon crawfordi (Diptera: Phoridae), a parasite of fire ants. Ann Entom Soc Am 80:148-151

Feener DH, Brown BV (1992) Reduced foraging of Solenopsis geminata (Hymenoptera: Formicidae) in the presence of parasitic Pseudacteon spp. (Diptera: Phoridae). Ann Entomol Soc Am 85:80-84

Feener DH, Jacobs LF, Schmidt JO (1996) Specialized parasitoid attracted to a pheromone of ants. Anim Behav 51:61-66

Gazal V, Bailez O, Viana-Bailez AM (2009) Mechanism of host recognition in Neodohrniphora elongata (Brown) (Diptera: Phoridae). Anim Behav 78:1177-1182 
Gilbert LE, Morrison LW (1997) Patterns of host specificity in Pseudacteon parasitoid flies (Diptera: Phoridae) that attack Solenopsis Fire ants (Hymenoptera: Formicidae). Environ Entomol 26:11491154

Hölldobler B (1999) Multimodal signals in ant communication. J Comput Phys A 184:129-141

Jackson BD, Morgan ED (1993) Insect chemical communication: pheromones and exocrine glands of ants. Chemoecology 4:125-144

LeBrun EG, Feener DH (2002) Linked indirect effects in ant-phorid interactions: impacts on ant assemblage structure. Oecologia 133:599-607

Lin BB (2007) Agroforestry management as an adaptive strategy against potential microclimate extremes in coffee agriculture. Agric For Meteorol 144:85-94

Maschwitz UA, Weissflog A, Seebaur S, Disney R, Witte V (2008) Studies on European ant decapitating flies (Diptera: Phoridae): I. Releasers and phenology of parasitism of Psuedacteon formicarum. Sociobiol 51:127-140

Moorehead SA, Feener DH (2000) Visual and chemical cues used in host location and acceptance by a dipteran parasitoid. J Insect Behav 14:613

Morrison LW, Gilbert LE (1999) Host specificity in two additional Pseudacteon spp. (Diptera: Phoridae), parasitoids of Solenopsis fire ants (Hymenoptera: Formicidae). Fla Entomol 82:404409

Morrison LW, King JR (2004) Host location behavior in a parasitoid of imported fire ants. J Insect Behav 17:367-383

Morrison LW, Porter SD (2006) Post-release host-specificity testing of Pseudacteon tricuspis, a phorid parasitoid of Solenopsis invicta fire ants. Biocontrol 51:195-205

Orr MR (1992) Parasitic flies (Diptera: Phoridae) influence foraging rhythms and caste division of labor in the leaf-cutter ant, Atta cephalotes (Hymenoptera: Formicidae). Behav Ecol Sociobiol 30:395-402

Orr MR, Seike SH, Benson WW, Gilbert LE (1995) Flies suppress fire ants. Nature 373:292-293

Orr MR, De Camargo RE, Benson WW (2003) Interactions between ant species increase arrival rates of an ant parasitoid. Anim Behav 65:1187-1193

Owens ED, Prokopy RJ (1986) Relationship between reflectance spectra of host plant surfaces and visual detection of host fruit by Rhgoletis promonella flies. Phys Entomol 11:297-307

Partan SR, Marler P (2002) Issues in the classification of multimodal communication signals. Am Nat 166:231-245

Perfecto I, Vandermeer J (2006) The effect of an ant-hemipteran mutualism on the coffee berry borer (Hypothenemus hampei) in southern Mexico. Agric Ecosyst Environ 117:218-221

Philpott SM (2005a) Ant patchiness: a spatially quantitative test in coffee agroecosystems. Naturwissenschaften 93:386-392

Philpott SM (2005b) Trail-mediated effects of parasitic phorid flies (Diptera: Phoridae) on ant (Hymenoptera: Formicidae) competition and resource access in coffee agroecosystems. Environ Entomol 24:1089-1094

Philpott SM, Maldonado J, Vandermeer J, Perfecto I (2004) Taking tropic cascades up a level: behaviorally-modified effects of phorid flies on ants and ant prey in coffee agroecosystems. Oikos 105:141-147

Philpott SM, Perfecto I, Vandermeer J, Uno S (2009) Spatial scale and density dependence in a hostparasitoid system: an arboreal ant, Azteca instabilis and its Pseudacteon phorid parasitoid. Environ Entomol 38:790-796

Plowes RM, LeBrun EG, Brown BV, Gilbert LE (2009) A Review of Pseudacteon (Diptera: Phoridae) that parasitize ants of the Solenopsis geminata complex (Hymenoptera: Formicidae). Ann Entomol Soc Am 102:937-958

Porter SD (1998) Biology and behavior of Pseudacteon decapitating flies (Diptera: Phoridae) that parasitize Solenopsis fire ants (Hymenoptera: Formicidae). Fla Entomol 81:292-309

Porter SD, Fowler HG, Campiolo S, Pesquero MA (1995) Host specificity of several Pseudacteon (Diptera: Phoridae) parasites of fire ants (Hymenoptera: Formicidae) in South America. Fla Entomol 78:70-75

Puckett RT, Calixto A, Barr CL, Harris M (2007) Sticky traps for monitoring Pseudacteon parasitoids of Solenopsis fire ants. Environ Entomol 36:584-588

Vandermeer J, Perfecto I, Nuñez GI, Philpott SM, Ballinas AG (2002) Ants (Azteca sp.) as potential biological control agents in shade coffee production in Chiapas, Mexico. Agroforest Syst 56:271-276

Vandermeer J, Perfecto I, Philpott SM (2008) Clusters of ant colonies and robust criticality in a tropical agroecosystem. Nature 451:457-460 
Vander Meer RK, Porter SD (2002) Fire ant, Solenopsis invicta worker alarm pheromones attract Pseudacteon phorid flies. Proc. 2002 Imported Fire Ant Conference, 24-26 March 2002, Athens, GA, p. 5-9

Wheeler JW, Blum MS, Torgerson RL (1975) Cyclopentyl kentones: Identification and function in Azteca ants. Science 183:254-255

Wuellner CT, Dall'Aglio-Holvercem CG, Benson WW, Gilbert LE (2002) Phorid fly (Diptera: Phoridae) ovipostion behavior and fire ant (Hymenoptera: Formicidae) reaction to attack differ according to phorid species. Ann Entomol Soc Am 95:257-266 